\title{
Evaluation of FIB-4 Index as a Marker for Hepatocellular Carcinoma in HIV - Hepatitis C Co-Infection
}

\section{Sobia Nizami', Jason Zucker ${ }^{2 *}$ and Shobha Swaminathan ${ }^{1}$}

${ }^{1}$ Rutgers New Jersey Medical School, Newark, USA

${ }^{2}$ Columbia University Medical Center, New York, USA

\begin{abstract}
Background: Chronic co-infection with hepatitis $\mathrm{C}(\mathrm{HCV})$ and HIV leads to progressive liver fibrosis and increases the risk for hepatocellular carcinoma $(\mathrm{HCC})$, creating the need for cancer surveillance. However, screening methods for HCC are controversial. We evaluated Fibrosis-4 (FIB-4); an index calculated from platelet count, alanine aminotransferase, aspartate aminotransferase and age; as a screening tool for HCC in patients with HIV-HCV coinfection.
\end{abstract}

Methods: This was a retrospective chart review of adult outpatients followed at our HIV clinic for the period 20082014, including 11 patients with HIV-HCV co-infection and HCC. Age- and sex-matched control groups were selected, including patients with co-infection without cirrhosis or HCC, HIV mono-infection; and patients with co-infection and cirrhosis without HCC. Mean FIB-4 indices were calculated for each group for comparison.

Results: The mean patient age was 55 years. Median CD4+ counts for the groups ranged from 413 to $552 / \mu \mathrm{L}$ $(p>0.05)$. The mean FIB-4 was not significantly different between patients with HIV (1.66 \pm 0.93$)$, compared to patients with HIV-HCV $(2.11 \pm 0.82)$. Mean FIB-4 score was significantly higher in patients with co-infection and cirrhosis $(6.5 \pm$ 5.1) compared to patients with co-infection without cirrhosis $(2.11 \pm 0.82 ; p=0.011)$. The mean FIB-4 score was higher for patients with co-infection and HCC, compared to patients with co-infection $(p=0.009)$, or HIV only $(p=0.004)$ without cancer. Mean FIB-4 score was not significantly different between patients with co-infection and cancer, and patients with co-infection and cirrhosis without cancer (5.87 \pm 4.22 and $6.5 \pm 5.1$ respectively).

Conclusion: FIB-4 appears to be a marker of cirrhosis but not specifically HCC in chronic HIV-HCV co-infection. FIB-4 can be a cost-effective strategy to identify patients with HIV and HCV who have advanced cirrhosis, and thereby are at increased risk for development of HCC.

Keywords: FIB-4; HIV; Hepatitis C; Hepatocellular carcinoma

\section{Background}

Cirrhosis and hepatocellular carcinoma (HCC) are a significant cause of mortality in human immunodeficiency virus (HIV) and hepatitis C (HCV) co-infected patients [1]. Liver disease in chronic hepatitis $\mathrm{C}$ infection progresses more rapidly in the setting of HIV co-infection, compared to HCV infection alone [2]. The prevalence of cirrhosis among patients with HIV-HCV co-infection has increased over the last 15 years and is estimated at $13 \%$, while the incidence of HCC is estimated to be $1.62 \%$ [1]. To screen for HCC occurrence and identify patients at highest risk, a number of non-invasive and costeffective techniques have been studied. The current recommendation from the American Association for the Study of Liver Diseases (AASLD) is to screen high-risk patients with liver ultrasound every 6 months [3]. However, there is only limited data to support this recommendation.

The Fibrosis-4 (FIB-4) is an index score calculated from platelet count, alanine aminotransferase (ALT), aspartate aminotransferase (AST), and age [2]. In prior studies, FIB-4 has been found to be a strong indicator for liver fibrosis in patients with chronic hepatitis B, HIV and hepatitis $C[4,5]$. A score of $<1.25$ excludes extensive fibrosis, and a score $>3.25$ is highly suggestive of extensive fibrosis [4]. There is also limited data suggesting that FIB-4 may be an independent marker for HCC [6]. In this study, we evaluated the utility of FIB-4 as a screening tool for HCC in patients co-infected with HIV and hepatitis C.

\section{Methods}

This retrospective chart review was performed at the Rutgers Infectious Diseases Practice at the University Hospital in Newark, New
Jersey. Approval was obtained from the Rutgers Institutional Review Board prior to study initiation. Adult patients undergoing regular outpatient treatment and follow-up for HIV at the clinic during the period 2008-2014 were included in the study. Patients with chronic active hepatitis B infection, alcoholic liver disease, and other conditions causing chronic liver disease were excluded. We included all patients with HIV and hepatitis C co-infection, who had been diagnosed with HCC. We then identified an equal number of age- and gender-matched patients to set up three control groups: persons with HIV infection alone, persons with HIV and hepatitis C co-infection without HCC and patients with HIV, hepatitis $\mathrm{C}$ and liver cirrhosis without HCC (due to the strong association of FIB-4 with cirrhosis). Data collected included patient demographics, AST and ALT levels, platelet count, CD4+count, HIV viral load, and hepatitis B status. For the study group of patients with HCC, data closest to the time of their HCC diagnosis was collected and for the control groups, laboratory values and data were taken from tests performed for routine outpatient surveillance. Mean FIB-4 indices were calculated for each group and compared for significant differences by analysis-of-variance.

*Corresponding author: Sobia Nizami, MD, Rutgers New Jersey Medical School 150 Bergen St, I-level 248 Newark, NJ 07103, USA, Tel: 973-972-1057; E-mail: sobia.nizami@rutgers.edu

Received April 07, 2016; Accepted April 25, 2016; Published May 02, 2016

Citation: Nizami S, Zucker J, Swaminathan S (2016) Evaluation of FIB-4 Index as a Marker for Hepatocellular Carcinoma in HIV - Hepatitis C Co-Infection. J AIDS Clin Res 7: 570. doi:10.4172/2155-6113.1000570

Copyright: (C) 2016 Nizami S, et al. This is an open-access article distributed under the terms of the Creative Commons Attribution License, which permits unrestricted use, distribution, and reproduction in any medium, provided the original author and source are credited. 


\section{Results}

A total of 11 patients were identified from the clinic population with HIV, hepatitis $\mathrm{C}$ and diagnosis of hepatocellular carcinoma who fulfilled the criteria to be included in the study. Nine of these patients had liver cirrhosis based on computed tomography imaging and/or liver biopsy. After random selection and matching for patient age and sex, eleven patients were included in the control group with HIV and hepatitis $\mathrm{C}$ without hepatocellular carcinoma, and 11 in the control group with HIV patients only. Nine patients were selected with HIV, hepatitis $\mathrm{C}$ and liver cirrhosis without evidence of hepatocellular carcinoma. A total of 42 patients were included in the study. Table 1 describes the demographics of the patients in the four groups. The mean patient age was 55.6 years, $20(45 \%)$ were male. The median $\mathrm{CD} 4+$ cell counts/ $\mu \mathrm{L}$ were not significantly different between the 4 groups and are shown in Figure 1. One patient in the control group of co-infection with cirrhosis without cancer, was positive for hepatitis B surface antigen. The remaining patients in the study were negative for hepatitis B infection.

The mean FIB-4 scores for the patients are shown in Figure 2. Mean FIB-4 was not significantly different between patients with HIV only (1.66 \pm 0.93$)$, compared to patients with HIV and Hepatitis C (2.11 $\pm 0.82)$. The mean FIB-4 score was $5.87( \pm 4.22)$ in the patients with HIV, hepatitis C co-infection and HCC, and $6.5( \pm 5.1)$ in the patients with co-infection and cirrhosis without cancer $(p>0.05)$. The mean FIB-4 score was significantly higher in patients with co-infection and cirrhosis $(6.5 \pm 5.1)$ compared with patients with co-infection without cirrhosis $(2.11 \pm 0.82 ; \mathrm{p}=0.011$, Figure 2$)$. The mean FIB-4 score was significantly higher for patients with co-infection and cancer, compared to patients with co-infection ( $\mathrm{p}=0.009$ ), or HIV only $(\mathrm{p}=0.004)$ without cancer.

The FIB-4 scores in the 2 patients with co-infection and HCC without cirrhosis were 1.29 and 2.76. Eight patients (73\%) of the

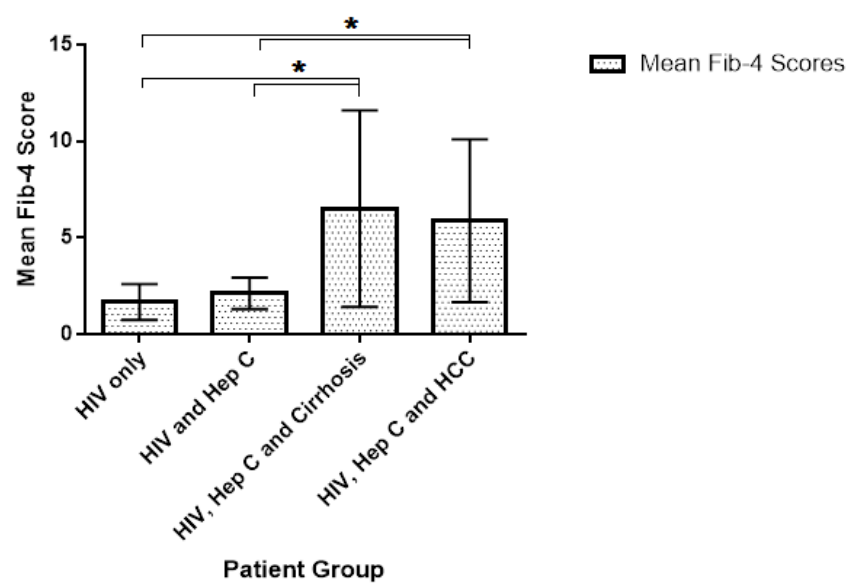

Figure 1: Median CD-4+ cell counts of patients in the four study groups.

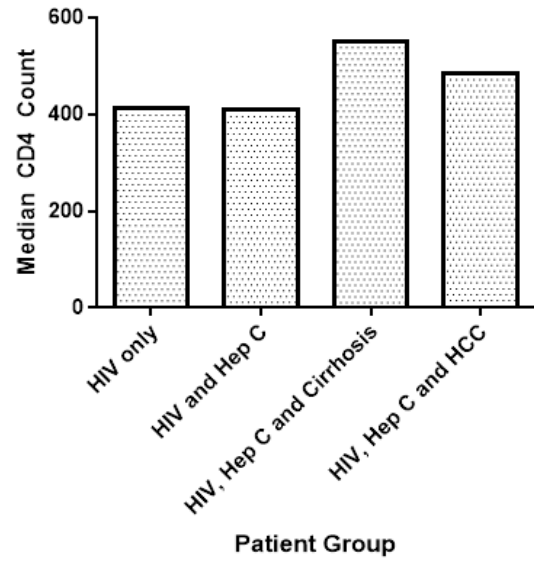

Median CD4 Count

Figure 2: Mean FIB-4 scores in the four groups. The mean values were compared for significant differences using analysis-of-variance ${ }^{*} p<0.05$

HCC cases were localized at the time of diagnosis and treated with chemoablation.

\section{Discussion}

Chronic infection with hepatitis $\mathrm{C}$ and HIV leads to progressive liver fibrosis and increases the risk for hepatocellular carcinoma, creating the need for cancer surveillance. Among persons infected with HIV, lower CD4+ cell counts increase the risk of HCC [7]. The gold standard for diagnosing cirrhosis is a liver biopsy, however concerns regarding risks from an invasive procedure lead to development of noninvasive tests to diagnose liver fibrosis. FIB-4 was proposed as a noninvasive marker to diagnose 3 levels of liver fibrosis by Sterling et al. [4]. It was reported to have an overall accuracy of $87 \%$. The advantage of FIB-4 over other non-invasive markers is the low cost, as it is calculated from routine laboratory parameters. It can be done at the bedside with minimal additional costs. The test was replicated in further studies and established as a routine screening test for liver fibrosis in patients with chronic liver disease. Subsequent studies also reported FIB-4 to be a strong indicator of liver cirrhosis $[8,9]$. Suh et al. reported that in patients with chronic hepatitis B infection, high FIB- 4 scores of $>2.4$ correlated with hepatocellular carcinoma incidence [10]. High FIB-4 was shown to be a strong predictor and risk factor for hepatocellular carcinoma in HIV-infected patients [6]. In our study, we assessed whether a higher FIB-4 could be associated with hepatocellular carcinoma in patients with HIV/hepatitis C co-infection. However, nine out of 11 patients in our study with hepatocellular carcinoma also had liver cirrhosis based on imaging. There was no significant difference in the FIB- 4 score in cirrhotic patients with, or without, HCC in the setting of co-infection. The mean FIB-4 score was significantly higher in patients with cirrhosis, than in patients without cirrhosis in co-infection.

Our study was a retrospective study with small numbers and it is possible that we may have been able to detect larger differences

\begin{tabular}{|c|c|c|c|c|}
\hline & $\begin{array}{l}\text { HIV only } \\
(n=11)\end{array}$ & $\begin{array}{l}\text { HIV-HCV co-infection } \\
(n=11)\end{array}$ & $\begin{array}{c}\text { HIV-HCV co-infection and liver } \\
\text { cirrhosis }(n=9)\end{array}$ & $\begin{array}{l}\text { HIV-HCV co-infection and } \\
\text { HCC * }(n=11)\end{array}$ \\
\hline Mean patient age & 55 & 55 & 57 & 55 \\
\hline Male $(\%)$ & 45 & 45 & 55 & 45 \\
\hline Black (\%) & 100 & 100 & 100 & 100 \\
\hline Median HIV Viral Load (copies/ml) & $<20$ & $<20$ & $<20$ & $<20$ \\
\hline
\end{tabular}

${ }^{*} 9$ out of 11 patients with HCC had liver cirrhosis. 
Citation: Nizami S, Zucker J, Swaminathan S (2016) Evaluation of FIB-4 Index as a Marker for Hepatocellular Carcinoma in HIV - Hepatitis C CoInfection. J AIDS Clin Res 7: 570. doi:10.4172/2155-6113.1000570

with a larger number of patients. The small sample size limited the determination of sensitivity, specificity or predictive values for FIB-4. However, the data does show that FIB-4 can be a cost-effective strategy to identify patients with HIV and HCV who may have advanced cirrhosis, and are thereby at increased risk for the development of HCC. In conclusion, our study shows FIB-4 to be a marker of cirrhosis but not specifically hepatocellular carcinoma in patients with chronic HIV and hepatitis C co-infection. More research is required to identify other biochmemical or molecular markers for early diagnosis of HCC.

\section{Declaration of Conflicts of Interest}

The authors have no conflicts of interest to declare.

\section{Funding}

This research received no specific grant from any funding agency in the public, commercial, or not-for-profit sectors.

\section{References}

1. Ioannou GN, Bryson CL, Weiss NS, Miller R, Scott JD, et al. (2013) The prevalence of cirrhosis and hepatocellular carcinoma in patients with human immunodeficiency virus infection. Hepatology 57: 249-257.

2. Sterling RK, Lissen E, Clumeck N, Sola R, Correa MC, et al. (2006) Development of a simple noninvasive index to predict significant fibrosis in patients with HIV HCV coinfection. Hepatology 43: 1317-1325.
3. Bruix J, Sherman M (2011) Management of hepatocellular carcinoma: An update. Hepatology 53: 1020-1022.

4. Vallet-Pichard A, Mallet V, Pol S (2006) FIB-4: A simple, inexpensive and accurate marker of fibrosis in HCV-infected patients. Hepatology 44: 769.

5. Berenguer J, Zamora FX, Aldámiz-Echevarría T, Von Wichmann MA, Crespo $\mathrm{M}$, et al. (2015) Comparison of the Prognostic Value of Liver Biopsy and FIB-4 Index in Patients Coinfected With HIV and Hepatitis C Virus. Clinical Infectious Diseases 60: 950-958.

6. Park LS, Tate JP, Justice AC, Lo Re V, Lim JK, et al. (2011) FIB-4 Index Is Associated with Hepatocellular Carcinoma Risk in HIV-Infected Patients. Cancer Epidemiology Biomarkers \& Prevention 20: 2512-2517.

7. Clifford GM, Rickenbach M, Polesel J, Dal Maso L, Steffen I, et al. (2008) Influence of HIV-related immunodeficiency on the risk of hepatocellular carcinoma. Aids 22: 2135-2141.

8. Trang T, Petersen JR, Snyder N (2008) Non-invasive markers of hepatic fibrosis in patients co-infected with HCV and HIV: Comparison of the APRI and FIB-4 index. Clinica Chimica Acta 397: 51-54.

9. Adler M, Gulbis B, Moreno C, Evrard S, Verset G, et al. (2008) The predictive value of FIB-4 versus FibroTest, APRI, Fibrolndex and Forns index to noninvasively estimate fibrosis in hepatitis $C$ and nonhepatitis $C$ liver diseases. Hepatology 47: 762-763.

10. Suh B, Park S, Shin DW, Yun JM, Yang H-K, et al. (2015) High liver fibrosis index FIB-4 is highly predictive of hepatocellular carcinoma in chronic hepatitis B carriers. Hepatology 61: 1261-1268. 